\title{
Validação da PANAS numa amostra portuguesa de pessoas idosas em resposta social
}

\section{PANAS validation in a Portuguese sample of older adults supported by social institutions}

\author{
Laura Lemos (1) \\ Helena Espírito-Santo \\ Cristiana Duarte-Figueiredo (1) \\ Diana Santos \\ Luís Cunha \\ Fernanda Daniel
}

(1) Centro Interdisciplinar de Investigação Psicossocial, Instituto Superior Miguel Torga, Portugal

(2) Centro de Investigação em Neuropsicologia e Intervenção Cognitivo-Comportamental, Faculdade de Psicologia e Ciências da Educação da Universidade de Coimbra, Portugal

(3) Centro de Estudos e Investigação em Saúde da Universidade de Coimbra, Portugal

Recebido: 12/11/2019; Revisto: 26/11/2019; Aceite: 27/11/2019.

https://doi.org/10.31211/rpics.2019.5.2.160

\section{Resumo}

Contexto: O afeto positivo e negativo são duas dimensões psicobiológicas do bem-estar subjetivo (BES) relevantes para a forma como é experienciada a circunstância da recurso a apoio institucional na vida de muitas pessoas idosas. Objetivo: O objetivo do estudo foi validar, no contexto de resposta social, uma versão breve da Positive and Negative Affect Schedule (PANAS), um questionário que permite avaliar o afeto positivo e negativo. Métodos: Numa amostra de 389 idosos (61 - 100 anos; $M=80,89$; $D P=7,48$ anos), avaliados através da PANAS, da Satisfaction With Life Scale e da Geriatric Depression Scale-8, testaram-se as propriedades psicométricas e realizou-se uma análise fatorial exploratória da PANAS. Numa segunda amostra de 383 sujeitos ( 60 - 99 anos; $M=80,27 ; D P=7,87$ anos) efetuou-se uma análise fatorial confirmatória. Resultados: Obteve-se uma versão reduzida da PANAS, com 14 itens, estrutura bidimensional, adequadas consistências internas, validade convergente e divergente para o Afeto Positivo/AP e Afeto Negativo/AN. A estabilidade temporal (intervalo $=1,44$ meses) foi igualmente adequada para o AP e para o AN $(p<0,001)$. A análise fatorial confirmatória revelou um ajustamento adequado para a estrutura bidimensional da PANAS-14 (AGFI = 0,91; CFI $=0,93 ;$ SRMR $=0,05 ;$ RMSEA = 0,06; PCLOSE = 0,12). Conclusão: A PANAS-14 é um instrumento breve psicometricamente adequado para a avaliação do PA e do NA em pessoas idosas em resposta social.

Palavras-Chave: Envelhecimento; Resposta Social; Bem-Estar Subjetivo; PANAS.

\section{DI\&D | ISMT \\ rpics@ismt.pt \\ https://rpics.ismt.pt}

Publicação em Acesso Aberto

(02019. O(s) Autor(es). Este é um artigo de acesso aberto distribuído sob a Licença Creative Commons Attribution, que permite uso, distribuição e reprodução sem restrições em qualquer meio, desde que o trabalho original seja devidamente citado

\author{
Laura Lemos \\ Instituto Superior Miguel Torga \\ Rua Augusta, 46 \\ 3000-061 Coimbra \\ Portugal \\ laurastlemos@gmail.com
}




\begin{abstract}
Background: Positive and negative affect are two psychobiological dimensions of subjective wellbeing (BES) relevant to the way institutional support is experienced in the lives of many older people. Aim: The study aimed to validate, in the context of institutional support, a brief version of the Positive and Negative Affect Schedule (PANAS), a questionnaire that allows us to evaluate positive and negative affect. Methods: A sample of 389 old adults (61- 100 years; $M=80.89$; $S D=$ 7.48 years) was assessed with the PANAS, Satisfaction With Life Scale, and Geriatric Depression Scale-8. In this sample, the psychometric properties of the PANAS were tested, and an exploratory factor analysis was performed. In a second sample of 383 subjects ( $60-99$ years; $M=80.27 ; S D=7.87$ years), confirmatory factor analysis was executed. Results: A reduced version of PANAS was obtained, with 14 items, a two-dimensional structure, adequate internal consistency, convergent, and divergent validity for the Positive Affect/PA and Negative Affect/NA. Temporal stability (interval = 1.44 months) was equally adequate for PA and NA $(p<0.001)$. Confirmatory factor analysis revealed an adequate adjustment for the twodimensional structure of PANAS-14 ( $A G F I=0.91 ; C F I=0.93 ; S R M R=0.05 ; R M S E A=0.06 ; P C L O S E=0.12)$. Conclusions: The PANAS-14 is a brief psychometrically suitable tool for the assessment of PA and NA in institutionalized older adults supported by social institutions.
\end{abstract}

Keywords: Aging; Social Institution; Subjective wellbeing; PANAS.

\title{
Introdução
}

A investigação sobre o envelhecimento tem evidenciado um interesse particular no Bem-Estar Subjetivo (BES). A preocupação sobre o possível impacto das alterações físicas, psicológicas e sociais que acompanham o processo de envelhecimento no BES das pessoas idosas (Diener, Scollon, \& Lucas, 2003), bem como o objetivo de promoção da saúde nesta etapa do ciclo vital, poderão explicar este movimento. De acordo com Galinha e Ribeiro (2005), o BES remete para uma dimensão positiva da saúde, associada a um envelhecimento saudável (Diener, 2000; Guedea et al., 2006), sendo a promoção desta dimensão nas pessoas idosas uma estratégia de saúde pública nos países desenvolvidos (Jivraj, Nazroo, Vanhoutte, \& Chandola, 2014).

Estudos recentes apontam para uma estabilidade do BES ao longo da vida, sugerindo que a idade não é um preditor de BES, pois se as condições de vida se mantiverem, se houver perceção de uma saúde e rede interpessoal satisfatórias, os níveis de BES tendem a não decrescer (Diener \& Lucas, 2000; Isaacowitz, 2005; Lima et al., 2001; Oliveira, Queiroz, \& Costa, 2012; Sposito, Diogo, Cintra, Neri, \& Guariento, 2010). Uma alteração significativa nas condições de vida das pessoas idosas poderá ser o recurso a instituições de apoio social. Por um lado, este apoio poderá constituir-se como essencial no acesso a cuidados de saúde e apoio social, no entanto, poderá também promover estados emocionais negativos, na medida em que a pessoa poderá ver-se confrontada com algum grau de dependência e fragilidade, sobretudo quando beneficiam da valência residencial. As instituições de apoio social, nomeadamente no que respeita ao seu ambiente físico, têm demonstrado influenciar a qualidade de vida e o bem-estar das pessoas idosas (Burton \& Sheehan, 2010). De acordo com a investigação, a perceção que a pessoa idosa tem do seu estado de saúde bem como a satisfação com a instituição onde reside são variáveis que interferem com o bem-estar e com a satisfação com a vida (Bockerman, Johansson, \& Saarni, 2012; Donnenwerth \& Petersen, 1992). Segundo Donnenwerth e Petersen (1992), a institucionalização terá um efeito positivo quando mediada pela perceção do idoso face à saúde, ainda que tenha um efeito negativo quando mediada pelo fator satisfação com a residência, o que implica um efeito negativo da variável institucionalização face ao bem-estar da pessoa idosa. Por outro lado, Bockerman et al. (2012) realizaram um estudo com população idosa finlandesa em que concluíram que quem vive em instituições apresenta maiores níveis satisfação com a vida. Desta forma e de acordo com Cardão (2009) a institucionalização 
nesta etapa do ciclo vital poderá apresentar vantagens e inconvenientes para a pessoa idosa. Por um lado, a institucionalização permite maior acessibilidade a serviços sociais e médicos que poderão ter impacto positivo na qualidade de vida do indivíduo e, por outro, implica a perda e a quebra dos laços familiares (Ferretti, Soccol, Albrecht, \& Ferraz, 2014), que influenciarão a adaptação positiva ao novo contexto (Cardão, 2009; Runcan, 2012). O recurso a outras valências institucionais poderá, também, ser um fator que influencia os níveis de BES uma vez que, por um lado, poderá pressupor algum nível de dependência e fragilidade em termos de saúde, e por outro, poderá fazer face a necessidades que de outra forma haveria maior dificuldade em serem supridas. A forma como a pessoa experiencia o apoio institucional parece assim ter impacto nos níveis de BES, legitimando a sua investigação com esta população.

O BES refere-se à avaliação subjetiva que as pessoas fazem das suas vidas, incluindo julgamentos cognitivos globais de satisfação e a experiência de emoções positivas e negativas (Diener, 1984; Diener et al., 2003). A investigação sobre o BES centra-se na forma como as pessoas vivem e experienciam as suas vidas, quer do ponto de vista cognitivo (Diener, 1984), caracterizado pela satisfação com a vida em domínios específicos (p. ex., a capacidade física e mental e os relacionamentos sociais), quer do ponto de vista afetivo, caracterizado pelo equilíbrio entre emoções positivas e negativas (Albuquerque \& Tróccoli, 2004; Diener, 2000; Diener, Suh, Lucas, \& Smith, 1999; Galinha \& Pais-Ribeiro, 2005). As emoções positivas estão relacionadas com experiências agradáveis subjetivas, traduzindo-se num estado no qual se sente entusiasmo e energia, das quais são exemplo a satisfação, a confiança e a felicidade (Crawford \& Henry, 2004). Ao invés, as emoções negativas refletem sofrimento e desconforto, caracterizando-se por vivências desagradáveis relativamente a distintas atividades do dia-a-dia (Watson, Clark, \& Tellegen, 1988), salientando-se, como exemplos, a tristeza e a culpa (Crawford \& Henry, 2004).

Assim, o afeto positivo e negativo são dimensões da experiência emocional e permitem diferenciar a ansiedade e a depressão. De acordo com o modelo tripartido de Clark e Watson (1991), a ansiedade é caracterizada por níveis elevados de ativação fisiológica, sendo a depressão caracterizada por diminuição do interesse, perda de energia e lentificação psicomotora (Laurent et al., 1999). Assim e tendo em conta as dimensões do afeto, sujeitos com ansiedade e depressão tendem a revelar níveis mais elevados de afeto negativo, e os sujeitos somente com sintomatologia depressiva apresentam também baixos níveis de afeto positivo.

As medidas de autorrelato são as mais utilizadas para avaliar os constructos que compõem o BES. Essas medidas são rápidas, de fácil aplicação e são sensíveis o suficiente para captar as diferentes componentes do bem-estar com bons níveis de confiabilidade e validade. A Positive and Negative Affect Schedule (PANAS; Watson et al., 1988) é um instrumento que permite avaliar as duas dimensões da afetividade, constituindo-se como uma medida breve, de fácil administração e válida que mede ambas as dimensões do afeto. Originalmente, a escala incluiu vinte itens, dez dos quais correspondentes ao Afeto Positivo e os restantes dez ao Afeto Negativo (Watson et al., 1988); a validação portuguesa da escala é constituída por vinte e dois itens, onze dos quais correspondentes ao Afeto Positivo e os restantes onze ao Afeto Negativo (Simões, 1993). Esta escala surgiu após estudos relativos à estrutura bidimensional do afeto e com o objetivo de desenvolvimento de uma escala reduzida, que fosse breve e de fácil administração, superando assim lacunas de escalas anteriores no que respeita às propriedades psicométricas, as quais tinham baixos valores de validade e fidedignidade (Watson et al., 1988).

Já no que respeita às versões portuguesas (Galinha \& Pais-Ribeiro, 2005; Simões, 1993), a PANAS foi validada tendo por base os mesmos pressupostos metodológicos adotados pelos autores da versão original, ainda que tendo em consideração as características peculiares que definem o afeto, bem como a disparidades lexicais, diferenças de contexto e cultura (Costa, 2013; Galinha \& Pais-Ribeiro, 2005; Simões, 1993). Desta forma, a escala portuguesa apresenta na sua generalidade boas propriedades psicométricas (Costa, 2013; Galinha \& Pais-Ribeiro, 2005; Simões, 1993) quando comparada com a validação da versão original americana, apresentando 13 de 22 itens similares em ambas as escalas (Costa, 
2013; Simões, 1993). A PANAS tem ainda sido alvo de validação para outros países e amostras. O Quadro 1 sintetiza a revisão da literatura dos estudos de validação da escala.

\section{Quadro 1}

Revisão da Literatura (continua)

\begin{tabular}{|c|c|c|c|c|c|}
\hline $\begin{array}{l}\text { Autor(es) } \\
\text { (Ano) }\end{array}$ & Amostra & $N^{\circ}$ Itens & $\begin{array}{l}\text { Consistência } \\
\text { interna }\end{array}$ & Teste-reteste & $\begin{array}{l}\text { Análise } \\
\text { Fatorial }\end{array}$ \\
\hline $\begin{array}{l}\text { Watson et al. } \\
\text { (1988) }\end{array}$ & $\begin{array}{c}N=663 \\
\text { Estudantes }\end{array}$ & 20 itens & $\begin{array}{l}A P=0,88 \\
A N=0,87\end{array}$ & $\begin{array}{l}A P=0,68 \\
A N=0,71\end{array}$ & $\begin{array}{c}2 \text { fatores } \\
\text { (AFE) }\end{array}$ \\
\hline $\begin{array}{l}\text { Kercher } \\
\text { (1992) }\end{array}$ & $\begin{array}{l}N=804 \\
\text { Idosos }\end{array}$ & 10 itens & $\begin{array}{l}A P=0,75 \\
A N=0,81\end{array}$ & - & $\begin{array}{l}2 \text { fatores } \\
\text { (AFC) }\end{array}$ \\
\hline $\begin{array}{l}\text { Simões } \\
\text { (1993) }\end{array}$ & $\begin{array}{c}N=226 \\
\text { Estudantes }\end{array}$ & 22 itens & $\begin{array}{l}A P=0,82 \\
A N=0,85\end{array}$ & - & - \\
\hline $\begin{array}{l}\text { Huebner \& Dew } \\
\text { (1995) }\end{array}$ & $\begin{array}{c}\mathrm{N}=266 \\
\text { Adolescentes }\end{array}$ & 10 itens & $\begin{array}{l}A P=0,85 \\
A N=0,84\end{array}$ & - & $\begin{array}{l}2 \text { fatores } \\
\text { (AFE) }\end{array}$ \\
\hline $\begin{array}{l}\text { Wilson Gullone, \& Moss } \\
\text { (1998) }\end{array}$ & $\begin{array}{c}\mathrm{N}=228 \\
\text { Crianças e } \\
\text { adolescentes }\end{array}$ & 20 itens & $\begin{array}{l}A P=0,78 \\
A N=0,81\end{array}$ & - & $\begin{array}{c}2 \text { fatores } \\
\text { (AFC) }\end{array}$ \\
\hline $\begin{array}{l}\text { Mackinnon et al. } \\
\text { (1999) }\end{array}$ & $\begin{array}{c}\mathrm{N}=2651 \\
\text { Comunidade }\end{array}$ & 10 itens & $\begin{array}{l}A P=0,78 \\
A N=0,87\end{array}$ & - & $\begin{array}{l}2 \text { fatores } \\
\text { (AFC) }\end{array}$ \\
\hline $\begin{array}{l}\text { Laurent et al. } \\
\text { (1999) }\end{array}$ & $\begin{array}{l}\mathrm{N}=707 \\
\text { Crianças }\end{array}$ & 30 itens & $\begin{array}{l}A P=0,89 \\
A N=0,94\end{array}$ & - & 2 fatores \\
\hline $\begin{array}{l}\text { Sandín et al. } \\
\text { (1999) }\end{array}$ & $\begin{array}{l}\mathrm{N}=712 \\
\text { Estudantes }\end{array}$ & 20 itens & $\begin{array}{l}A P=0,87 \\
A N=0,89\end{array}$ & - & 2 fatores \\
\hline $\begin{array}{l}\text { DePaoli \& Sweeney } \\
(2000)\end{array}$ & $\begin{array}{c}\mathrm{N}=69 \\
\text { Estudantes }\end{array}$ & 20 itens & $\begin{array}{l}A P=0,85 \\
A N=0,86\end{array}$ & - & - \\
\hline $\begin{array}{l}\text { Crawford \& Henry } \\
(2004)\end{array}$ & $\begin{array}{c}\mathrm{N}=1003 \\
\text { Adultos } \\
\text { comunidade }\end{array}$ & 20 itens & $\begin{array}{l}A P=0,89 \\
A N=0,85\end{array}$ & - & $\begin{array}{c}2 \text { fatores } \\
\text { (AFC) }\end{array}$ \\
\hline $\begin{array}{l}\text { Galinha \& Pais-Ribeiro } \\
(2005)\end{array}$ & $\begin{array}{c}N=348 \\
\text { Estudantes }\end{array}$ & 20 itens & $\begin{array}{l}A P=0,86 \\
A N=0,89\end{array}$ & - & $\begin{array}{l}2 \text { fatores } \\
\text { (AFE) }\end{array}$ \\
\hline $\begin{array}{l}\text { Thompson } \\
\text { (2007) }\end{array}$ & $\begin{array}{c}N=407 \\
\text { Estudantes }\end{array}$ & 10 itens & $\begin{array}{l}\mathrm{AP}=0,78 \\
\mathrm{AN}=0,76\end{array}$ & $\begin{array}{l}A P=0,84 \\
A N=0,84\end{array}$ & $\begin{array}{l}2 \text { fatores } \\
\text { (AFC) }\end{array}$ \\
\hline $\begin{array}{l}\text { Lim, Yu, Kim, \& Kim } \\
(2010)\end{array}$ & $\begin{array}{c}N=218 \\
\text { Amostra clínica }\end{array}$ & 20 itens & $\begin{array}{l}A P=0,87 \\
A N=0,91\end{array}$ & $\begin{array}{l}\text { AP }=0,79 \\
A N=0,89\end{array}$ & $\begin{array}{c}2 \text { fatores } \\
\text { (AFC) }\end{array}$ \\
\hline
\end{tabular}

Nota. $\mathrm{AP}=$ Afeto Positivo; NA = Afeto Negativo; $A F E=$ Análise fatorial exploratória; $A F C=$ Análise fatorial confirmatória; EAP = Afeto positivo na amostra de estudantes; EAN = Afeto Negativo na amostra de estudantes; PCAP = Afeto Positivo na amostra da população clínica; PCAN = Afeto Negativo na amostra da população clínica.

a Este estudo está inserido no Projeto Trajetórias do Envelhecimento. 


\section{Quadro 1}

Revisão da Literatura (continuação)

\begin{tabular}{|c|c|c|c|c|c|}
\hline $\begin{array}{l}\text { Autor(es) } \\
\text { (Ano) }\end{array}$ & Amostra & $N^{\circ}$ Itens & $\begin{array}{l}\text { Consistência } \\
\text { interna }\end{array}$ & Teste-reteste & $\begin{array}{l}\text { Análise } \\
\text { Fatorial }\end{array}$ \\
\hline $\begin{array}{l}\text { Gyollai, Köteles, \& } \\
\text { Demetrovics } \\
\text { (2011) }\end{array}$ & $\begin{array}{c}\quad N=1629 \\
\text { Estudantes (E) e } \\
\text { População clínica } \\
\text { (PC) }\end{array}$ & 20 itens & $\begin{aligned} E A P & =0,73 \\
E A N & =0,79 \\
P C A P & =0,65 \\
P C A P & =0,67\end{aligned}$ & - & $\begin{array}{l}2 \text { fatores } \\
\text { (AFC) }\end{array}$ \\
\hline $\begin{array}{l}\text { Karim, Weisz, \& Rehman } \\
\text { (2011) }\end{array}$ & $\begin{array}{c}N=423 \\
\text { Estudantes }\end{array}$ & 10 itens & $\begin{array}{l}A P=0,75 \\
A N=0,80\end{array}$ & - & $\begin{array}{l}2 \text { fatores } \\
\text { (AFC) }\end{array}$ \\
\hline $\begin{array}{l}\text { Cotigã } \\
(2012)\end{array}$ & $\begin{array}{l}\quad N=120 \\
\text { Funcionários }\end{array}$ & 11 itens & $\begin{array}{c}\mathrm{AP}=0,83 \\
\mathrm{AN}=0,80 \\
\text { Total }=0,81\end{array}$ & $\begin{array}{l}A P=0,39 \\
A N=0,43\end{array}$ & - \\
\hline $\begin{array}{l}\text { Dufey \& Fernandez } \\
\text { (2012) }\end{array}$ & $\begin{array}{c}N=437 \\
\text { Estudantes }\end{array}$ & 20 itens & $\begin{array}{l}\mathrm{AP}=0,71 \\
\mathrm{AN}=0,82\end{array}$ & $\begin{array}{l}A P=0,65 \\
A N=0,77\end{array}$ & $\begin{array}{l}2 \text { fatores } \\
\text { (AFE) }\end{array}$ \\
\hline $\begin{array}{l}\text { Costa } \\
(2013) a\end{array}$ & $\begin{array}{l}N=555 \\
\text { Idosos }\end{array}$ & 20 itens & $\begin{array}{l}A P=0,79 \\
A N=0,84\end{array}$ & $\begin{array}{l}A P=0,45 \\
A N=0,10\end{array}$ & $\begin{array}{l}2 \text { fatores } \\
\text { (AFE) }\end{array}$ \\
\hline $\begin{array}{l}\text { Carvalho et al. } \\
(2013)\end{array}$ & $\begin{array}{c}N=3728 \\
\text { Comunidade }\end{array}$ & 20 itens & $\begin{array}{l}A P=0,88 \\
A N=0,87\end{array}$ & - & $\begin{array}{c}2 \text { fatores } \\
\text { (AFE) }\end{array}$ \\
\hline $\begin{array}{l}\text { Galinha, Pereira, \& Esteves } \\
(2014)\end{array}$ & $\begin{array}{c}N=836 \\
\text { Estudantes }\end{array}$ & 10 itens & $\begin{array}{l}A P=0,86 \\
A N=0,89\end{array}$ & $\begin{array}{c}\text { Invariância } \\
\text { intemporal x } 2\end{array}$ & $\begin{array}{l}2 \text { fatores } \\
\text { (AFC) }\end{array}$ \\
\hline $\begin{array}{l}\text { Nolla, Queral, \& Miró } \\
\text { (2014) }\end{array}$ & $\begin{array}{l}N=436 \\
\text { Idosos }\end{array}$ & 20 itens & $\begin{array}{l}A P=0,91 \\
A N=0,90\end{array}$ & - & $\begin{array}{l}2 \text { fatores } \\
\text { (AFE) }\end{array}$ \\
\hline $\begin{array}{l}\text { Buz, Pérez-Arechaederra, } \\
\text { Fernández-Pulido, \& Urchaga } \\
\text { (2015) }\end{array}$ & $\begin{array}{l}N=585 \\
\text { Idosos }\end{array}$ & 20 itens & $\begin{array}{l}A P=0,93 \\
A N=0,83\end{array}$ & - & $\begin{array}{c}2 \text { fatores } \\
\text { (AFE) }\end{array}$ \\
\hline $\begin{array}{l}\text { Sousa, Marques-Vieira, } \\
\text { Severino, Pozo Rosado, \& José } \\
\text { (2016) }\end{array}$ & $\begin{array}{l}N=171 \\
\text { Doença renal } \\
\text { crónica }\end{array}$ & 20 itens & $\begin{array}{l}A P=0,87 \\
A N=0,88\end{array}$ & Total $=0,91$ & $\begin{array}{c}2 \text { fatores } \\
\text { (AFE) }\end{array}$ \\
\hline $\begin{array}{l}\text { Humboldt \& Leal } \\
\text { (2017) }\end{array}$ & $\begin{array}{c}N=1291 \\
\text { Idosos comunidade }\end{array}$ & 20 itens & $\begin{array}{l}A P=0,92 \\
A N=0,88\end{array}$ & - & 2 fatores \\
\hline
\end{tabular}

Nota $. \mathrm{AP}=$ Afeto positivo; $\mathrm{NA}=$ Afeto Negativo; $A F E=$ Análise fatorial exploratória; $A F C=$ Análise fatorial confirmatória; $\mathrm{EAP}=$ Afeto Positivo na amostra de estudantes; EAN = Afeto Negativo na amostra de estudantes; PCAP = Afeto Positivo na amostra da população clínica; PCAN = Afeto Negativo na amostra da população clínica. a Este estudo está inserido no Projeto Trajetórias do Envelhecimento.

Em suma, as pessoas idosas têm de lidar com desafios físicos, mentais e sociais particulares. Um desses desafios é o apoio prestado por instituições que surge frequentemente como resposta à solidão e às dificuldades físicas, mentais ou neurocognitivas. A forma como a pessoa idosa experiencia o apoio 
institucional poderá influenciar as dimensões do afeto positivo e negativo, as componentes emocionais do BES. Assim, é de relevo que este segmento da população seja estudado na sua dimensão afetiva.

A PANAS foi validada em várias populações e culturas e existe um estudo preliminar com pessoas idosas sob resposta social realizado pelo nosso grupo de investigação (Costa, 2013). No entanto, os estudos com pessoas idosas que beneficiam de apoio institucional são limitados e havia que estender o estudo prévio a amostras maiores. Adicionalmente, versões breves de questionários são um aspeto importante para evitar sobrecarregar uma população idosa que caracteristicamente apresenta baixa escolarização, presença de sintomatologia mental, neurocognitiva e física e idade avançada (e.g., Daniel, Vicente, Guadalupe, Silva, \& Espirito-Santo, 2015; Espirito-Santo \& Daniel, 2018; Fastame \& Cavallini, 2011; Fastame, Hitchcott, Penna, \& Murino, 2016; Figueiredo-Duarte, Espirito-Santo, Sério, Marques, \& Daniel, 2019; Runcan, 2012; Vicente et al., 2014).

Assim, o presente estudo teve como objetivos: 1) determinar a estrutura fatorial da PANAS numa amostra de pessoas em resposta social de idade avançada e verificar se é possível derivar dessa análise uma versão mais curta; 2 ) estudar as propriedades psicométricas da PANAS na mesma amostra (Estudo 1); 3) efetuar uma análise fatorial confirmatória noutra amostra de pessoas idosas que beneficiam de apoio institucional (Estudo 2).

\section{Métodos}

\section{Participantes}

A amostra utilizada na presente investigação foi distinta consoante o estudo (Estudo 1 e Estudo 2). Contudo, para ambos os estudos, a amostra foi selecionada de acordo com os seguintes critérios: sujeitos com idade igual ou superior a 60 anos em resposta social, tendo sido excluídos os sujeitos com dificuldades de compreensão e expressão verbal (determinadas na entrevista inicial e confirmadas nos registos médicos dos utentes). Após a seleção, os participantes foram divididos aleatoriamente pelos dois grupos de cada estudo. Metade da amostra, utilizada no Estudo 1, incluiu 389 idosos e a outra metade abrangeu 383 idosos incluídos no Estudo 2.

\section{Instrumentos}

Questionário sociodemográfico. Com este questionário recolheu-se informação sobre o sexo, idade, estado civil, profissão, nível de escolaridade, área geográfica e tipo de resposta social.

Lista de Afetos Positivos e Negativos. A Positive Affect and Negative Affect Schedule (PANAS, versão original de Watson et al., 1988; versão portuguesa de Simões, 1993) é uma escala que permite avaliar a afetividade e o bem-estar subjetivo, constituída por 22 itens, divididos em igual número quanto pelas subescalas/fatores Afeto Positivo e Negativo. A PANAS compreende um formato de resposta tipo Likert de cinco pontos, variando a sua pontuação entre 11 e 55 pontos (nível mais alto de afeto) em cada subescala.

Escala de Depressão Geriátrica de 8 itens. A Geriatric Depression Scale-8 (GDS, versão original de Yesavage et al., 1983; tradução para a população portuguesa de Barreto, Leuschner, Santos, \& Sobral, 2007; validação de Figueiredo-Duarte et al., 2019) é uma escala que objetiva o rastreio da sintomatologia depressiva na população geriátrica institucionalizada (Figueiredo-Duarte et al., 2019). Esta versão da GDS é constituída por oito itens, variando a sua pontuação entre 0 e 8 (mais sintomas depressivos). O valor de alfa de Cronbach da versão original foi de 0,94 , tendo o valor da população portuguesa sido de 0,87 . No que respeita à presente investigação, o valor de alfa de Cronbach encontrado foi de 0,85 .

Escala de Satisfação com a Vida. A Satisfaction With Life Scale (SWLS, versão original de Diener, Emmons, Larsen e Griffin, 1985; versão portuguesa de Simões, 1992) avalia a satisfação dos sujeitos com a vida, no que respeita ao bem-estar subjetivo dos mesmos. A escala compreende cinco itens, com 
opção de resposta de cinco pontos numa escala de Likert, variando a sua pontuação entre os 5 e os 25 pontos (Simões, 1992). O valor de confiabilidade da versão portuguesa numa amostra prévia de pessoas idosas sob resposta social do Projeto Trajetórias do Envelhecimento foi de 0,76 (Costa, 2013), tendo sido de 0,78 no presente estudo.

\section{Procedimentos}

A presente investigação está inserida no projeto Trajetórias do Envelhecimento do Instituto Superior Miguel Torga, tendo para os devidos efeitos sido elaborados pedidos de autorização aos autores das escalas integrantes do protocolo, bem como às instituições e respetivos utentes através de consentimento informado. O protocolo foi administrado por avaliadores treinados de acordo com os objetivos do estudo, tendo integrado um questionário sociodemográfico, a PANAS, a SWLS e a GDS-8. Para análise da estabilidade temporal da escala o protocolo foi administrado num segundo momento a uma subamostra de 120 pessoas (1,44 meses de intervalo). Todos os questionários foram lidos em voz alta para padronizar a administração e minimizar eventuais dificuldades de compreensão das questões.

\section{Estudo 1: Análise Fatorial Exploratória}

\section{Participantes}

A amostra incluiu 389 sujeitos, com idades compreendidas entre os 61 e os 100 anos e uma média de idades de 81 anos $(D P=7,48)$. Esta amostra foi constituída por $289(74,3 \%)$ sujeitos do sexo feminino e $100(25,7 \%)$ do sexo masculino. No que respeita ao estado civil, a amostra foi constituída por 43 $(11,1 \%)$ sujeitos solteiros, 21 (5,4\%) sujeitos separados/ divorciados, 243 (62,5\%) viúvos e $82(21,1 \%)$ casados ou em união de facto. De acordo com a ocupação prévia/profissão, $340(88,1 \%)$ sujeitos tinham uma profissão manual, tendo os restantes $46(11,9 \%)$ uma profissão de índole intelectual. Quanto ao nível de escolaridade, 118 (30,3\%) sujeitos não eram escolarizados, sendo que 49 (12,6\%) dos sujeitos sabiam ler e escrever ainda que não tivessem escolaridade; $168(43,2 \%)$ completou o $1 .^{\circ}$ ciclo do ensino básico; 30 (7,7\%) concluiu o $2 .^{\circ}$ ciclo do ensino básico; tendo os restantes $24(6,2 \%)$ concluído o ensino secundário. No que respeita à resposta social, dez $(2,6 \%)$ dos sujeitos frequentavam o centro de convívio; 211 (54,9\%) estavam inseridos em regime de centro de dia; $12(3,1 \%)$ frequentavam o centro de noite; 147 (38,3\%) residiam em estruturas residenciais para idosos; estando os restantes 4 (1\%) integrados no serviço de apoio domiciliário. A amostra deste estudo contou com $152(39,1 \%)$ de participantes provenientes de meio urbano, sendo os restantes 237 (60,9\%) provenientes de meio rural.

\section{Análise Estatística}

A análise estatística no Estudo 1 foi realizada com recurso ao programa IBM SPSS Statistics (v.25), tendo sido utilizada estatística paramétrica tendo em conta o critério proposto por Kim (2013), que, para amostras com mais de 300 participantes, propõe que valores absolutos de assimetria inferiores a 2 e de curtose inferiores a 4 sejam indicadores de não desvio da normalidade. Inicialmente realizou-se uma análise fatorial exploratória por forma a analisar a estrutura fatorial da escala, bem como a adequabilidade dos seus constituintes. De seguida, utilizou-se a análise paralela de Monte Carlo com o intuito de confirmar a solução fatorial rodada (Varimax e Quartimax) mais adequada. Seguidamente foram inspecionados os valores de fidedignidade da escala através do valor de alfa de Cronbach, bem como os valores de alfa se item excluído no sentido de corroborar a estrutura fatorial que anteriormente se salientou. Para finalizar, foi ainda importante verificar os valores da escala em estudo no que respeita à sua validade convergente e divergente com outros instrumentos, através dos valores de correlação de Pearson e do teste $t$ de Student para amostras emparelhadas. 


\section{Tabela 1}

Caracterização Sociodemográfica da Amostra para o Estudo $1(N=389)$

\begin{tabular}{|c|c|c|c|}
\hline \multicolumn{2}{|c|}{ Variáveis sociodemográficas } & \multirow{2}{*}{$\frac{n}{289}$} & \multirow{2}{*}{$\begin{array}{c}\% \\
74,3\end{array}$} \\
\hline Soyo & Feminino & & \\
\hline sexo & Masculino & 100 & 25,7 \\
\hline \multirow{4}{*}{$\begin{array}{l}\text { Faixa Etária } \\
(M=81,00 ; \\
D P=7,48)\end{array}$} & $61-70$ & 36 & 9,3 \\
\hline & $71-80$ & 129 & 33,2 \\
\hline & $81-90$ & 191 & 49,1 \\
\hline & $91-100$ & 33 & 8,5 \\
\hline \multirow{4}{*}{ Estado Civil } & Solteiro(a) & 43 & 11,1 \\
\hline & Casado(a) & 82 & 21,1 \\
\hline & Divorciado(a) & 21 & 5,4 \\
\hline & Viúvo(a) & 243 & 62,5 \\
\hline \multirow{2}{*}{ Profissão } & Manual & 340 & 88,1 \\
\hline & Intelectual & 46 & 11,9 \\
\hline \multirow{5}{*}{$\begin{array}{l}\text { Nível de } \\
\text { Escolaridade }\end{array}$} & Sem escolaridade & 118 & 30,3 \\
\hline & Sabe ler e escrever & 49 & 12,6 \\
\hline & $1^{\circ}$ ciclo & 168 & 43,2 \\
\hline & $2^{\circ}$ ciclo & 30 & 7,7 \\
\hline & Ensino secundário & 24 & 6,2 \\
\hline \multirow{5}{*}{$\begin{array}{l}\text { Resposta } \\
\text { Social }\end{array}$} & Centro de convívio & 10 & 2,6 \\
\hline & Centro de dia & 211 & 54,9 \\
\hline & Centro de noite & 12 & 3,1 \\
\hline & Estrutural residencial & 147 & 38,3 \\
\hline & Apoio domiciliário & 4 & 1,0 \\
\hline \multirow{2}{*}{ Área geográfica } & Rural & 237 & 60,9 \\
\hline & Urbano & 152 & 39,1 \\
\hline
\end{tabular}

Nota. $M=$ Média; $D P=$ Desvio Padrão; $n=$ Número de sujeitos.

\section{Resultados}

Análise fatorial exploratória. A PANAS de 22 itens foi submetida a uma análise fatorial exploratória de componentes principais não rodada e rodada com rotação Varimax e Quartimax por forma a verificar a sua estrutura após maximização das suas saturações. Em consequência, a escala apresentou um total de quatro fatores com uma variância total explicada de 49,56, bem como um valor de Keyser Meyer Olkin $(K M O)$ de 0,87 e um teste de esfericidade de Bartlett com significância estatística ( $p$ < 0,001). Contudo, a análise paralela de Monte Carlo (22 itens $\times 389$ sujeitos) indicou valores de critério para os eigenvalues que conduziram à decisão de reter apenas dois fatores. Desta forma, a análise fatorial exploratória foi novamente realizada forçada a dois fatores, tendo apresentado uma variância total explicada de 38,89. Após a análise não rodada ter obtido itens com saturações em ambos os fatores, aplicámos a rotação tanto Quartimax como Varimax. As soluções foram idênticas, verificandose que os Itens 8 (Agressivo) e 13 (Envergonhado) apresentavam saturações muito baixas no fator Afeto Negativo, pelo que este critério levou à exclusão dos mesmos. Por forma a obter uma versão reduzida, mas que fosse balanceada (mesmo número de itens positivos e negativos), tiveram de ser retidos 
somente itens que tivessem um valor de saturação superior a 0,63 no respetivo fator (saturações muito boas explicativas de 40\% da variância; Hair, Anderson, Tatham, \& Black, 1995; Pearson \& Hall, 1993). Desta forma, excluíram-se do fator Afeto Positivo os Itens 1 (Interessado), 10 (Orgulhoso), 17 (Atencioso) e 21 (Emocionado), e os Itens 4 (Aborrecido) e 6 (Culpado) do fator Afeto Negativo. Por fim, repetida a análise com a exclusão dos itens referidos, a PANAS-14 apresentou-se como uma escala bifatorial, com uma variância total explicada de 48,64 (KMO =0,85; Teste de esfericidade de Bartlett: $p<0,001)$.

\section{Tabela 2}

Valores de Saturação dos Itens da PANAS- 14 nos Dois Fatores (Afeto Positivo e Afeto Negativo) ( $N=$ 389)

\begin{tabular}{|c|c|c|}
\hline Item (\# versão original) & Afeto Positivo & Afeto Negativo \\
\hline 1. Aflito (2) & - & 0,67 \\
\hline 2. Estimulado (animado) (3) & 0,70 & - \\
\hline 3. Forte (5) & 0,65 & - \\
\hline 4. Assustado (7) & - & 0,77 \\
\hline 5. Entusiasmado (arrebatado) (9) & 0,70 & - \\
\hline 6. Irritável (11) & - & 0,73 \\
\hline 7. Atento (12) & 0,61 & - \\
\hline 8. Inspirado (14) & 0,67 & - \\
\hline 9. Nervoso (15) & - & 0,77 \\
\hline 10. Decidido (16) & 0,63 & - \\
\hline 11. Inquieto (18) & - & 0,71 \\
\hline 12. Ativo (19) & 0,67 & - \\
\hline 13. Medroso (20) & - & 0,64 \\
\hline 14. Magoado (22) & - & 0,75 \\
\hline
\end{tabular}

Consistência interna. Os valores de consistência interna foram calculados através do valor de alfa de Cronbach para as subescalas do Afeto Positivo e Negativo. No que respeita ao Afeto Positivo o valor de alfa de Cronbach foi de 0,78, tendo o valor da subescala do Afeto Negativo sido de 0,84 . Quando inspecionados os valores de alfa-se-item-eliminado, foi possível verificar que nenhum dos valores de alfa dos itens se sobrepunha ao valor de alfa da escala total, o que corroborou a estrutura fatorial da escala.

Validades convergente e divergente. As validades convergente e divergente foram calculadas através de correlações de Pearson com os totais das escalas GDS-8 e SWLS. Quanto ao Afeto Positivo, no que respeita à validade convergente, foi encontrado um valor de correlação de 0,44 $(R 2 \times 100=19,4 \% ; p<$ $0,01)$ com a SWLS. No que respeita à validade divergente, as pontuações do Afeto Positivo apresentaram um valor de correlação de -0,24 ( $R 2 \times 100=5,8 \% ; p<0,01)$ com a GDS-8 e de -0,17 ( $R 2 \times 100=2,9 \%$; $p<0,01)$ com subescala de Afeto Negativo. Relativamente ao Afeto Negativo, no que respeita à validade 
convergente, foi encontrado um valor de correlação de 0,61 ( $R 2 \times 100=37,2 \% ; p<0,01)$ com a GDS-8 e um valor de $-0,34(R 2 \times 100=11,6 \% ; p<0,01)$ com a SWLS no que respeita à validade divergente.

Tabela 3

Correlações de Pearson entre o Afeto Positivo, Afeto Negativo da PANAS, GDS e SWLS (N=389)

\begin{tabular}{lcccc}
\hline Instrumentos & $\mathbf{1}$ & $\mathbf{2}$ & $\mathbf{3}$ & $\mathbf{4}$ \\
\hline 1. Afeto Positivo & - & & \\
2. Afeto Negativo & $-0,17^{* *}$ & - & \\
3. GDS & $-0,24^{* *}$ & $0,61^{* *}$ & - & \\
4. SWLS & $0,44^{* *}$ & $-0,34^{* *}$ & $-0,34^{* *}$ & - \\
\hline
\end{tabular}

Nota. PANAS = Positive and Negative Affect Schedule; GDS = Geriatric Depression Scale; SWLS = Satisfaction With Life Scale. ${ }^{*} p<0,05 ;{ }^{* *} p<0,01$.

Estabilidade temporal. Quando analisados os resultados da análise $t$ de Student para amostras emparelhadas foi possível depreender que no que concerne ao Afeto Positivo houve uma diminuição das pontuações entre os primeiro $(M=19,08 ; D P=4,79)$ e o segundo $(M=18,11 ; D P=5,78)$ momentos de avaliação, ainda que esta diferença não fosse estatisticamente significativa $[t(65)=1,49 ; p=0,15]$. Quanto ao Afeto Negativo foi também possível depreender, através da mesma análise, a diminuição das pontuações entre o primeiro $(M=17,64 ; D P=6,79)$ e o segundo $(M=16,05 ; D P=5,43)$ momentos, o que também não foi estatisticamente significativo $[t(65)=1,81 ; p=0,08]$. Por fim, a PANAS-14 apresentou as pontuações que se descrevem junto com a sua conversão numa escala uniforme de 0-100 baseada na percentagem do máximo possível (POMP) de acordo com a fórmula de Cohen, Cohen, Aiken e West (2010): MPOMP = [M - pontuação mínima possível (7)] / [pontuação máxima possível (35) - pontuação mínima possível (7)] x 100. No Afeto Positivo, a média foi de 19,66 (DP = 5,71; $\min =7,00 ;$ máx $=35 ; M P O M P=45,2 \%)$ e no Afeto Negativo, a média foi de 16,75 $(D P=6,96 ; \min =$ 7,00; máx = 35; MPOMP = 34,8\%). Como se pode observar, a percentagem da média foi superior para o Afeto Positivo.

\section{Estudo 2: Análise Fatorial Confirmatória}

\section{Participantes}

A amostra foi constituída por 383 sujeitos, com idades compreendidas entre os 60 e os 99 anos e uma média de idades de 80 anos ( $D P=7,87)$. Relativamente à caracterização da amostra, esta constituiu-se por $289(75,5 \%)$ mulheres e $94(24,5 \%)$ homens. No que respeita ao estado civil, a amostra ficou constituída por $47(12,3 \%)$ sujeitos solteiros, 34 (8,9\%) sujeitos separados/ divorciados, 222 (58,1\%) viúvos e 79 (20,7\%) casados ou em união de facto. De acordo com a ocupação prévia/profissão, 325 $(86,7 \%)$ sujeitos tinham uma profissão manual, tendo os restantes $50(13,3 \%)$ uma profissão de índole intelectual. Quanto ao nível de escolaridade, 101 (26,4\%) sujeitos não eram escolarizados, sendo que $56(14,6 \%)$ sabiam ler e escrever, ainda que não tivessem escolaridade; 183 (47,8\%) completou o $1 .^{\circ}$ ciclo do ensino básico; 26 (6,8\%) concluiu o $2 .^{\circ}$ ciclo do ensino básico; tendo os restantes 17 (4,4\%) concluído o ensino secundário. No que respeita à resposta social oito $(2,1 \%)$ dos sujeitos frequentavam o centro de convívio; 204 (53,5\%) dos sujeitos estavam inseridos em regime de centro de dia; 15 (3,9\%) frequentavam o centro de noite; $143(37,5 \%)$ residiam em estruturas residenciais para idosos; estando os restantes 11 (2,9\%) integrados no serviço de apoio domiciliário. A amostra deste estudo contou com 137 (35,5\%) participantes provenientes de meio urbano, sendo os restantes 247 (64,5\%) provenientes de meio rural. 
Tabela 4

Caracterização Sociodemográfica da Amostra do Estudo $2(\mathrm{~N}=383)$

\begin{tabular}{|c|c|c|c|}
\hline \multicolumn{2}{|c|}{ Variáveis sociodemográficas } & \multirow{2}{*}{$\frac{N}{289}$} & \multirow{2}{*}{$\frac{\%}{75,5}$} \\
\hline Sexo & Feminino & & \\
\hline & Masculino & 94 & 24,5 \\
\hline Faixa Etária & $61-70$ & 47 & 12,3 \\
\hline \multirow[t]{3}{*}{$(M=80 ; D P=7,87)$} & $71-80$ & 133 & 34,9 \\
\hline & $81-90$ & 175 & 45,9 \\
\hline & $91-100$ & 25 & 6,8 \\
\hline \multirow[t]{4}{*}{ Estado Civil } & Solteiro(a) & 47 & 12,3 \\
\hline & Casado(a) & 79 & 20,7 \\
\hline & Divorciado(a) & 34 & 8,9 \\
\hline & Viúvo(a) & 222 & 58,1 \\
\hline \multirow[t]{2}{*}{ Profissão } & Manual & 325 & 86,7 \\
\hline & Intelectual & 50 & 13,3 \\
\hline \multirow[t]{5}{*}{ Nível de Escolaridade } & Sem escolaridade & 101 & 26,4 \\
\hline & Sabe ler e escrever & 56 & 14,6 \\
\hline & $1^{\circ}$ ciclo & 183 & 47,8 \\
\hline & $2^{\circ}$ ciclo & 26 & 6,8 \\
\hline & Ensino secundário & 17 & 4,4 \\
\hline \multirow[t]{5}{*}{ Resposta Social } & Centro de convívio & 8 & 2,1 \\
\hline & Centro de dia & 204 & 53,5 \\
\hline & Centro de noite & 15 & 3,9 \\
\hline & Estrutural residencial & 143 & 37,5 \\
\hline & Apoio domiciliário & 11 & 2,9 \\
\hline \multirow[t]{2}{*}{ Proveniência } & Rural & 247 & 64,5 \\
\hline & Urbano & 137 & 35,5 \\
\hline
\end{tabular}

Nota. $M=$ Média; $D P=$ Desvio Padrão; $n=$ Número de sujeitos.

\section{Análise Estatística}

A análise estatística relativa ao Estudo 2 foi realizada através da utilização do programa IBM SPSS AMOS (v.24). Por forma a avaliar o ajustamento dos dados ao modelo de dois fatores da PANAS-14 itens, foi conduzida uma Análise fatorial confirmatória (AFC). Quanto à qualidade de ajustamento do modelo, esta foi avaliada tendo por base os seguintes indicadores: Qui-quadrado normalizado ( $\left.\chi_{2} / g l\right)$, Comparative Fit Index (CFI), Adjusted Goodness of Fit Index (AGFI), Standardized Root Mean Square Residual (SRMR), Root Mean Square Error of Approximation (RMSEA), e valor de $p$ para o teste da hipótese nula de que o RMSEA é inferior a 0,05 (PCLOSE), sendo seguidas as recomendações de Hu e Bentler (1999).

\section{Resultados}

Análise fatorial confirmatória. Os resultados obtidos para os indicadores do modelo de análise fatorial confirmatória, apresentados na Tabela 5, revelaram um ajustamento adequado. 


\section{Tabela 5}

Limites para Valores de Ajustamento de Modelos AFC (Hu \& Bentler, 1999)

\begin{tabular}{lll}
\hline Indicadores & Valores do Modelo & Valores de Referência \\
\hline $\mathbf{X} 2 / \boldsymbol{g l}$ & 2,28 & $<3$ bom; $<5$ aceitável \\
$\boldsymbol{C F I}$ & 0,93 & $>0,95$ excelente; $>0,90$ tradicional; \\
$\boldsymbol{A G F I}$ & 0,91 & $>0,80$ \\
SRMR & 0,05 & $<0,09$ \\
$\boldsymbol{R} M S E \boldsymbol{A}$ & 0,06 & $<0,05$ bom; $0,05-0,10$ moderado; $>0,10$ mau \\
$\boldsymbol{P C L O S E}$ & 0,12 & $>0,05$ \\
\hline
\end{tabular}

A representação gráfica do modelo, com os pesos de regressão estandardizados e com o valor de correlação entre as variáveis Afeto Positivo e Afeto Negativo pode ser inspecionada na Figura 1. Todos os valores foram estatisticamente significativos, embora a correlação entre as variáveis latentes que representam os tipos de afeto fosse fraca.

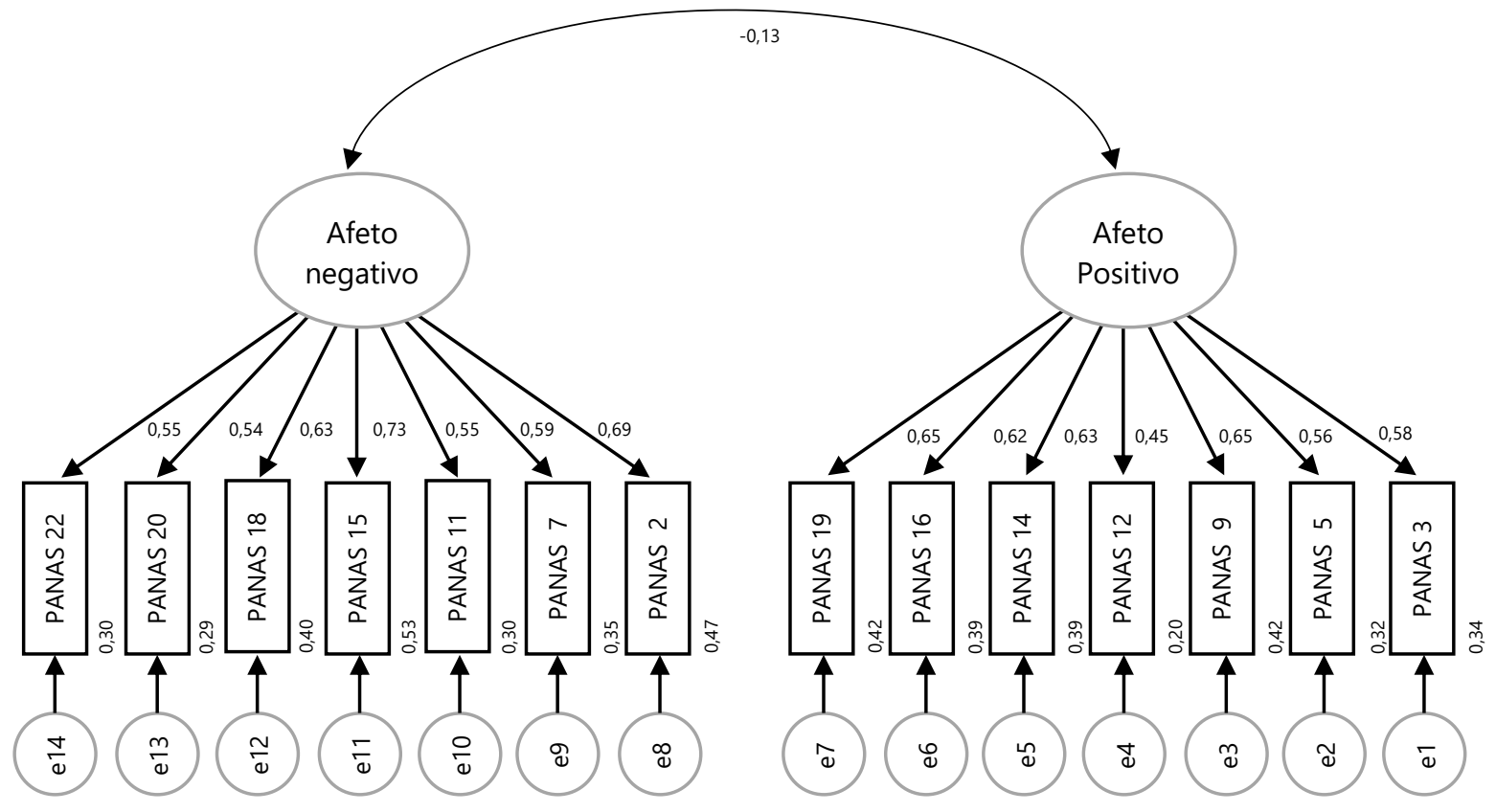

Figura 1. Modelo de análise fatorial confirmatória para o PANAS, com duas variáveis latentes, cada uma medida por sete indicadores. A correlação entre as subescalas Afeto Positivo e Afeto Negativo aparece representada na seta curva, à esquerda. Todos os valores foram significativos $(p<0,05)$. PANAS $2=$ Aflito; PANAS $3=$ Estimulado; PANAS 5 = Forte; PANAS $7=$ Assustado; PANAS $9=$ Entusiasmado; PANAS $11=$ Irritável; PANAS $12=$ Atento; PANAS $14=$ Inspirado; PANAS $15=$ Nervoso; PANAS $16=$ Decidido; PANAS $18=$ Inquieto; PANAS $19=$ Ativo; PANAS $20=$ Medroso; PANAS $22=$ Magoado .

Consistência interna. O valor de alfa de Cronbach para a consistência interna encontrado para as subescalas do Afeto Positivo e Afeto Negativo foi de 0,79 e de 0,81, respetivamente.

Nesta amostra, a PANAS-14 apresentou os seguintes valores: no Afeto Positivo, a média foi de 19,53 $(D P=5,71 ; \min =7,00 ;$ máx $=35 ;$ MPOMP $=44,8 \%)$ e no Afeto Negativo, a média foi de 16,36 ( $D P=$ 6,45; $\min =7,00 ;$ máx $=34 ;$ Мромр $=33,4 \%$ ). Como se pode observar, também nesta amostra a percentagem da média foi superior no Afeto Positivo. 


\section{Discussão}

Este trabalho integra dois estudos com o objetivo de validar a Escala de Afetos Positivos e Negativos numa amostra de pessoas de idade avançada que frequentam instituições de apoio social. $O$ trabalho forneceu evidência de que a versão portuguesa da PANAS é aceitável psicometricamente para avaliar pessoas idosas em contexto institucional.

Apesar de existirem validações portuguesas deste instrumento na sua versão integral (Costa, 2013; Galinha \& Pais-Ribeiro, 2005; Simões, 1993; Sousa et al., 2016) e uma proposta de uma versão reduzida (Galinha et al., 2014), o presente estudo obteve uma versão reduzida psicometricamente adequada para administrar a pessoas de idade avançada sob resposta social. Larsen e Diener (1992) defenderam que a PANAS não seria um instrumento adequado para pessoas idosas, pois nesta faixa etária a dimensão positiva e negativa do afeto estariam substancialmente relacionadas. No entanto, Kercher (1992), o primeiro investigador a usar este instrumento com pessoas mais velhas, concluiu que os dois fatores se mantêm relativamente independentes, confirmado por outros autores posteriormente (Buz, PérezArechaederra, Fernández-Pulido, \& Urchaga, 2015; Costa, 2013; Hillerås, Jorm, Herlitz, \& Winblad, 1998; Kercher, 1992). Ora, o nosso trabalho reforça essa ideia da independência do afeto positivo e do afeto negativo em pessoas idosas, contrariando a defesa de Larsen e Diener (1992).

O Estudo 1 apresenta a análise fatorial exploratória que conduziu à eliminação de quatro itens de cada uma das subescalas que compõem a PANAS, Afeto Positivo [Itens 1 (Interessado), 10 (Orgulhoso), 17 (Atencioso) e 21 (Emocionado)] e Afeto Negativo (Itens 4 (Aborrecido), 6 (Culpado), 8 (Hostil), 13 (Envergonhado)], partindo da versão portuguesa de Simões (1993) de 22 itens. A PANAS-14 passa a integrar um total de 14 itens mantendo os dois fatores encontrados noutros estudos (Galinha et al., 2014; Simões, 1993; Thompson, 2007; Watson et al., 1988). A subescala Afeto Positivo é composta pelos itens Estimulado/Animado, Forte, Entusiasmado, Atento, Inspirado, Decidido e Ativo e a subescala Afeto Negativo integra os itens Aflito, Assustado, Irritável, Nervoso, Inquieto, Medroso e Magoado. Alguns dos itens escolhidos para integrar a PANAS-14 diferem de outras propostas de versões reduzidas (Galinha et al., 2014; Kercher, 1992; Thompson, 2007), o que poderá dever-se a diferenças na escala integral que serviu de partida ao estudos, a questões culturais e linguísticas, mas também à especificidade das populações alvo das diferentes validações.

Os valores de consistência interna da PANAS-14 revelam-se adequados e semelhantes aos das validações prévias com populações diferentes (Carvalho et al., 2013; Cotigă, 2012; Crawford \& Henry, 2004; DePaoli \& Sweeney, 2000; Dufey \& Fernandez, 2012; Galinha \& Pais-Ribeiro, 2005; Galinha et al., 2014; Gyollai et al., 2011; Huebner \& Dew, 1995; Humboldt \& Leal, 2017; Karim et al., 2011; Laurent et al., 1999; Lim et al., 2010; Mackinnon et al., 1999; Sandín et al., 1999; Simões, 1993; Sousa et al., 2016; Thompson, 2007; Watson et al., 1988; Wilson et al., 1998) e aos de validações com pessoas idosas (Buz et al., 2015; Kercher, 1992) que frequentam instituições de apoio social (Costa, 2013).

No que diz respeito à validade convergente, encontramos correlações positivas fortes e significativas entre o Afeto Positivo e a SWLS, o que permite corroborar a ideia de que maiores níveis de Afeto Positivo se correlacionam com uma maior satisfação com a vida em pessoas idosas (Buz et al., 2015; Costa, 2013). O Afeto Negativo apresentou uma correlação positiva forte com a GDS-8, o que vem apoiar a relação de estados emocionais negativos e sintomatologia depressiva (Buz et al., 2015; Costa, 2013; Crawford \& Henry, 2004), confirmando que a subescala negativa mede o que se propõe a medir.

Relativamente à validade divergente, verificamos valores de correlação também fortes e significativos entre o Afeto Negativo e a SWLS, mas no sentido inverso, resultado que vem fortalecer outros estudos desenvolvidos com a mesma população (Buz et al., 2015; Costa, 2013). O Afeto Positivo encontra-se também inversamente correlacionado com a GDS-8, estando também em consonância com estudos prévios com pessoas idosas (Buz et al., 2015; Costa, 2013; Crawford \& Henry, 2004). 
A associação entre as duas dimensões da PANAS é significativa e negativa, resultado esperado, uma vez que se propõem a medir constructos opostos.

A estabilidade temporal foi verificada através da correlação teste-reteste, verificando-se uma correlação estatisticamente significativa entre os dois momentos de avaliação, embora com um valor inferior ao da validação de Watson et al. (1988) e ao da validação da versão reduzida de Thompson (2007). No entanto, as amostras destes dois estudos eram mais jovens, o que pode explicar uma maior manutenção no estado dos afetos ou até uma maior lembrança das respostas dadas no primeiro momento.

O Estudo 2 apresenta a análise fatorial confirmatória, tendo-se verificado que a PANAS reduzida a 14 itens retém os dois fatores (i. e., Afeto Positivo e Afeto Negativo) numa amostra de pessoas idosas sob resposta social. A estrutura de dois fatores demonstrou adequação aceitável para uma amostra desta natureza.

Assim, o nosso primeiro estudo aponta para uma versão mais curta da PANAS e o segundo estudo confirma a sua redução, acrescentando-se a algumas investigações que indicam a utilidade e importância de usar versões mais reduzidas (Cotigă, 2012; Galinha et al., 2014; Huebner \& Dew, 1995; Karim et al., 2011; Mackinnon et al., 1999; Thompson, 2007).

Apesar de não ser objetivo desta investigação, analisaram-se as médias e as percentagens do máximo possível em ambos os estudos, constatando-se que foram similares para os dois tipos de afeto, tendo o Afeto Positivo revelado valores superiores nas duas amostras. Comparando com outros estudos para os quais foi possível 1 efetuar o cálculo da percentagem do máximo possível (Bishop et al., 2011: AP = 49,5\% e AN = 22,5\%; Buz et al., 2015: AP = 57,6\% e AN = 23,8\%; Kercher, 1992: AP = 21,3\% e AN = 11,3\%; Nolla et al., 2014: $A$ Phomens $=35 \%$, APmulheres $=36 \%$ e ANhomens $=27,3 \%$, ANmulheres $=32,2 \%$; Ready et al., 2011: $\mathrm{AP}=60,7 \%$ e AN $=17,2 \%$, pode observar-se que os níveis de Afeto Positivo são superiores aos de Afeto Negativo, em consonância com o presente estudo. No entanto, esses estudos foram realizados com pessoas de idade avançada da comunidade e com maiores níveis de escolaridade. A presença de maiores níveis de AP permite-nos hipotetizar sobre a possibilidade de as pessoas de idade avançada sob resposta social terem desenvolvido uma boa capacidade de regulação emocional (Buz et al., 2015).

\section{Limitações}

Algumas limitações são de apontar a este estudo. Primeiro, apesar de as amostras serem numericamente expressivas, não deixaram de ser amostras de conveniência de voluntários, pelo que a natureza da amostra pode ter influenciado os resultados. A replicação será uma necessidade para determinar se estes resultados não são exclusivos das amostras presentes neste estudo.

Segundo, o baixo nível de escolaridade pode ter comprometido a compreensão dos itens da PANAS. Ainda assim, todo os questionários foram lidos em voz alta, pelo que se terá obviado eventuais dificuldades de compreensão.

Terceiro, há limitações na análise fatorial confirmatória. Por exemplo, a invariância dos fatores não foi analisada para as subamostras (e.g., sexo, estado civil, área geográfica, etc.). Desta forma, futuras pesquisas devem avaliar a influência potencial das variáveis sociodemográficas.

\section{Conclusão}

A PANAS-14 é um instrumento adequado para a avaliação do afeto positivo e negativo em pessoas idosas que frequentam instituições de apoio social, apresentando-se como um instrumento vantajoso pela sua menor dimensão e pela consequente redução do tempo de administração. Adicionalmente, os nossos

\footnotetext{
1 O estudo de Humboldt e Leal (2017) não apresentou médias e o estudo de Costa (2013) não fez parte da comparação pois estava integrado no Projeto Trajetórias do Envelhecimento.
} 
resultados apoiam a ideia de que o afeto é um indicador chave do BES das pessoas idosas institucionalizadas. Assim, a avaliação do afeto positivo e negativo em pessoas idosas sob resposta social poderá constituir-se como um recurso importante na compreensão holística destes sujeitos e no desenvolvimento de estratégias de intervenção que promovam o BES. Por fim, considerando as vantagens de um instrumento de avaliação breve sugere-se que estudos futuros investiguem a PANAS-14 de acordo com a Teoria da Resposta ao Item por forma a obter-se uma avaliação compreensiva e precisa das características dos itens (e.g., Pires, Filgueiras, Ribas, \& Santana, 2013).

Conflito de interesses | Conflict of interest: nenhum | none.

Fontes de financiamento | Funding sources: nenhuma | none.

Contributos: LL: Contributo significativo na redação e revisão do manuscrito. HES: Contributo significativo no desenvolvimento do desenho metodológico, supervisão da recolha de dados, análise estatística, redação e revisão do manuscrito. CFD: Contributo significativo na redação do manuscrito e tratamento dos dados. DS: Contributo significativo na redação do manuscrito e tratamento dos dados. LC: Contributo significativo na análise estatística e na revisão do manuscrito.

FD: Contributo significativo na análise estatística e na revisão do manuscrito.

\section{Referências}

Albuquerque, A. S., \& Tróccoli, B. T. (2004). Desenvolvimento de uma escala de bem-estar subjetivo [Development of a subjective wellness scale]. Psicologia: Teoria e Pesquisa, 20(2), 153-164. https://doi.org/10.1590/S0102-37722004000200008

Barreto, J., Leuschner, A., Santos, F., \& Sobral, M. (2007). Escala de Depressão Geriátrica [Geriatric Depression Scale]. In Grupo de estudos de envelhecimento cerebral e demência (Eds.), Livro de escalas e testes na demência (2nd ed., pp. 65-67). Lisboa: Novartis.

Bishop, A. J., Martin, P., Poon, L., \& Johnson, M. A. (2011). Exploring positive and negative affect as key indicators of life satisfaction among centenarians: Does cognitive performance matter? Journal of Aging Research, 2011, 1-10. https://doi.org/10.4061/2011/953031

Bockerman, P., Johansson, E., \& Saarni, S. I. (2012). Institutionalisation and subjective wellbeing for old-age individuals: Is life really miserable in care homes? Ageing and Society, 32(Part 7), 1176-1192. https://doi.org/10.1017/S0144686X1100081X

Burton, E., \& Sheehan, B. (2010). Care-home environments and well-being: Identifying the design features that most affect older residents. Journal of Architectural and Planning Research, 27(3), 237-256. Retrieved from www.jstor.org/stable/43030908.

Buz, J., Pérez-Arechaederra, D., Fernández-Pulido, R., \& Urchaga, D. (2015). Factorial structure and measurement invariance of the PANAS in Spanish older adults. The Spanish Journal of Psychology, 18(3), 113. https://doi.org/10.1017/sjp.2015.6

Cardão, S. (2009). O Idoso institucionalizado [The institutionalized elderly]. Lisboa: Coisas de Ler.

Carvalho, H. W. de, Andreoli, S. B., Lara, D. R., Patrick, C. J., Quintana, M. I., Bressan, R. A., ... Jorge, M. R. (2013). Structural validity and reliability of the Positive and Negative Affect Schedule (PANAS): Evidence from a large Brazilian community sample. Brazilian Journal of Psychiatry, 35(2), 169-172. https://doi.org/10.1590/1516-4446-2012-0957

Clark, L. A., \& Watson, D. (1991). Tripartite model of anxiety and depression: Psychometric evidence and taxonomic implications. Journal of Abnormal Psychology, 100(3), 316-336. https://doi.org/10.1037//0021843x.100.3.316

Cohen, P., Cohen, J., Aiken, L. S., \& West, S. G. (2010). The problem of units and the circumstance for POMP. Multivariate Behavioral Research, 34(3), 315-346. https://doi.org/10.1207/S15327906MBR3403_2 
Costa, A. (2013). Bem-estar subjetivo: Validação das escalas PANAS e SWLS a uma amostra de idosos portugueses institucionalizados [Subjective well-being: Validation of PANAS and SWLS scales for a sample of institutionalized Portuguese elderly] (Master's Thesis, Instituto Superior Miguel Torga). Retrieved from http://repositorio.ismt.pt/handle/123456789/318

Cotigă, M. I. (2012). Development and validation of a Romanian version of the expanded version of Positive and Negative Affect Schedule (PANAS-X). Procedia - Social and Behavioral Sciences, 33, 248-252. https://doi.org/10.1016/j.sbspro.2012.01.121

Crawford, J. R., \& Henry, J. D. (2004). The Positive and Negative Affect Schedule (PANAS): Construct validity, measurement properties and normative data in a large non-clinical sample. British Journal of Clinical Psychology, 43(3), 1-22. https://doi.org/10.1348/0144665031752934

Daniel, F., Vicente, H., Guadalupe, S., Silva, A., \& Espirito-Santo, H. M. A. (2015). Propriedades psicométricas da versão portuguesa do Inventário Geriátrico de Ansiedade numa amostra de idosos utentes de estruturas residenciais [Psychometric properties of the Portuguese Version of the Geriatric Anxiety Inventory in a sample of eldery people in residential care]. Revista Portuguesa de Investigação Comportamental e Social, 1(2), 15-30. https://doi.org/10.7342/ismt.rpics.2015.1.2.22

DePaoli, L. C., \& Sweeney, D. C. (2000). Further validation of the Positive and Negative Affect Schedule. Journal of Social Behavior and Personality, 15(4), 561-568.

Diener, E. (1984). Subjective well-being. Psychological Bulletin, 95(3), 542-575. Retrieved from http://labs.psychology.illinois.edu/ ediener/Documents/Diener_1984.pdf

Diener, E. (2000). Subjective well-being: The science of happiness and a proposal for a national index. American Psychologist, 55(1), 34-43. https://doi.org/10.1037/0003-066X.55.1.34

Diener, E., Emmons, R. A., Larsen, R. J., \& Griffin, S. (1985). The Satisfaction With Life Scale. Journal of Personality Assessment, 49(1), 71-75. https://doi.org/10.1207/s15327752jpa4901_13

Diener, E., \& Lucas, R. F. (2000). Subjective emotional well being. In M. Lewis \& J. M. Haviland-Jones (Eds.), Handbook of emotions (2nd ed., pp. 325-337). New York: Guilford.

Diener, E., Scollon, C. N., \& Lucas, R. E. (2003). The evolving concept of subjective well-being: The multifaceted nature of happiness. Advances in Cell Aging and Gerontology, 15, 187-2019. https://doi.org/10.1016/S15663124(03)15003-1

Diener, E., Suh, E. M., Lucas, L., R. E., \& Smith, H. L. (1999). Subjective well-being: Three decades of progress. Psychological Bulletin, 125(2), 276-302. https://doi.org/10.1037/0033-2909.125.2.276

Donnenwerth, G. V., \& Petersen, L. R. (1992). Institutionalization and well-being among the elderly. Sociological Inquiry, 62(4), 437-449. https://doi.org/10.1111/j.1475-682X.1992.tb00293.x

Dufey, M., \& Fernandez, A. M. (2012). Validez y confiabilidad del Positive Affect and Negative Affect Schedule (PANAS) en estudiantes universitarios chilenos. Revista Iberoamericana de Diagnóstico y Evaluación, 34(1), 157-173. Retrieved from https://www.aidep.org/03_ridep/R34/ART\%208.pdf

Espirito-Santo, H., \& Daniel, F. (2018). Optimism and well-being among institutionalized older adults. GeroPsych: The Journal of Gerontopsychology and Geriatric Psychiatry, 31(1), 5-16. https://doi.org/10.1024/1662-9647/a000182

Fastame, M. C., \& Cavallini, E. (2011). Working memory functions in healthy elderly people: The impact of institutionalization and advancing age on mnestic efficiency. Clinical Gerontologist, 34(3), 207-219. https://doi.org/10.1080/07317115.2011.555909

Fastame, M. C., Hitchcott, P. K., Penna, M. P., \& Murino, G. (2016). Does institutionalization influence perceived metamemory, psychological well-being, and working-memory efficiency in Italian elders? A preliminary study. Journal of Clinical Gerontology and Geriatrics, 7(1), 6-11. https://doi.org/10.1016/j.jcgg.2015.07.001

Ferretti, F., Soccol, B. F., Albrecht, D. C., \& Ferraz, L. (2014). Viver a velhice em ambiente institucionalizado [Living elderly in an institutionalized environment]. Estudos Interdisciplinares sobre o Envelhecimento, 19(2), 423437. Retrieved from http://seer.ufrgs.br/index.php/RevEnvelhecer/article/view/42378/32755 
Figueiredo-Duarte, C., Espirito-Santo, H., Sério, C., Marques, M., \& Daniel, F. (2019). Validity and reliability of a shorter version of the Geriatric Depression Scale in institutionalized older Portuguese adults (Manuscript accepted for publication). Aging and Mental Health. https://doi.org/10.1080/13607863.2019.1695739

Galinha, I. C., \& Pais-Ribeiro, J. L. (2005). Contribuição para o estudo da versão portuguesa da Positive and Negative Affect Schedule (PANAS): I-Abordagem teórica ao conceito de afecto [Contribution to the study of the Portuguese version of the Positive and Negative Affect Schedule (PANAS): I-Theoretical approach to the concept of affection]. Análise Psicológica, 2(22), 219-227. Retrieved from http://www.scielo.mec.pt/scielo.php?script=sci_abstract\&pid=S0870$82312005000200011 \&$ lng $=$ pt\&nrm=iso

Galinha, I. C., Pereira, C. R., \& Esteves, F. (2014). Versão reduzida da escala portuguesa de afeto positivo e negativo - PANAS-VRP: Análise fatorial confirmatória e invariância temporal [Portuguese version of the positive and negative affect scale - PANAS-VRP: Confirmatory factor analysis and temporal invariance]. Psicologia, 28(1), 53-65. Retrieved from http://www.scielo.mec.pt/scielo.php?script=sci_arttext\&pid=S0874-20492014000100005

Galinha, I., \& Ribeiro, J. L. P. (2005). História e evolução do conceito de bem-estar subjectivo [History and evolution of the concept of subjective well-being]. Psicologia, Saúde \& Doenças, 6(2), 203-214. Retrieved from https://pdfs.semanticscholar.org/f4c6/087802586c24274a7f70126092f90e088614.pdf

Guedea, M. T. D., Albuquerque, F. J. B. de, Tróccoli, B. T., Noriega, J. A. V., Seabra, M. A. B., \& Guedea, R. L. D. (2006). Relação do bem-estar subjetivo, estratégias de enfrentamento e apoio social em idosos [Relationship of subjective well-being, coping strategies and social support in the elderly]. Psicologia: Reflexão e Crítica, 19(2), 301-308. https://doi.org/10.1590/S0102-79722006000200017

Gyollai, A., Simor, P., Köteles, P., \& Demetrovics, Z. (2011). Psychometric properties of the Hungarian version of the original and the short form of the Positive and Negative Affect Schedule (PANAS). Neuropsychopharmacologia Hungarica, 13(2), 73-79. Retrieved from http://mpptnph.hu/images/gyollai.pdf

Hair, J. F., Jr., Anderson, R. E., Tatham, R. L., \& Black, W. C. (1995). Multivariate data analysis with readings (4th ed.). Upper Saddle River, NJ: Prentice-Hall, Inc.

Hillerås, P. K., Jorm, A. F., Herlitz, A., \& Winblad, B. (1998). Negative and positive affect among the very old: A survey on a sample age 90 years or older. Research on Aging, 20(5), 593-610. https://doi.org/10.1177/0164027598205003

Hu, Li-tze, \& Bentler, P. M. (1999). Cutoff criteria for fit indexes in covariance structure analysis: Conventional criteria versus new alternatives. Structural Equation Modeling: A Multidisciplinary Journal, 6(1), 1-55. https://doi.org/10.1080/10705519909540118

Huebner, E. S., \& Dew, T. (1995). Preliminary validation of the Positive and Negative Affect Schedule with adolescents. Journal of Psychoeducational Assessment, 13(3), 286-293. https://doi.org/10.1177/073428299501300307

Humboldt, S. V., \& Leal, I. (2017). Validation of a measure of positive and negative affect for use with cross national older adults. European Psychiatry, 41, S666. https://doi.org/10.1016/j.eurpsy.2017.01.1133

Isaacowitz, D. M. (2005). Correlates of well-being in adulthood and old age: A tale of two optimisms. Journal of Research in Personality, 39(2), 224-244. https://doi.org/10.1016/j.jrp.2004.02.003

Jivraj, S., Nazroo, J., Vanhoutte, B., \& Chandola, T. (2014). Aging and subjective well-being in later life. The Journals of Gerontology Series B: Psychological Sciences and Social Sciences, 69(6), 930-941. https://doi.org/10.1093/geronb/gbu006

Karim, J., Weisz, R., \& Rehman, S. U. (2011). International positive and negative affect schedule short-form (IPANAS-SF): Testing for factorial invariance across cultures. Procedia - Social and Behavioral Sciences, 15, 2016-2022. https://doi.org/10.1016/j.sbspro.2011.04.046

Kercher, K. (1992). Assessing subjective well-being in the old-old: The PANAS as a measure of orthogonal dimensions of positive and negative affect. Research on Aging, 14(2), 131-168. https://doi.org/10.1177/0164027592142001 
Kim, H.-Y. (2013). Statistical notes for clinical researchers: Understanding standard deviations and standard errors. Restorative Dentistry \& Endodontics, 38(4), 263-265. https://doi.org/10.5395/rde.2013.38.4.263

Larsen, R. J., \& Diener, E. (1992). Promises and problems with the circumplex model of emotion. In M. S. Clark (Ed.), Review of Personality and Social Psychology: Vol. 13. Emotion (pp. 25-59). Thousand Oaks, CA: Sage Publications, Inc.

Laurent, J., Catanzaro, S. J., Joiner Jr., T. E., Rudolph, K. D., Potter, K. I., Lambert, S., ... Gathright, T. (1999). A measure of positive and negative affect for children: Scale development and preliminary validation. Psychological Assessment, 11(3), 326-338. https://doi.org/10.1037/1040-3590.11.3.326

Lim, Y.-J., Yu, B.-H., Kim, D.-K., \& Kim, J.-H. (2010). The Positive and Negative Affect Schedule: Psychometric properties of the Korean version. Psychiatry Investigation, 7(3), 163-169. https://doi.org/10.4306/pi.2010.7.3.163

Lima, M. P., Simões, A., Vieira, C. C., Oliveira, A. L., Ferreira, J. A., Pinheiro, M. R. M. M., \& Matos, A. P. M. (2001). $O$ bem-estar subjetivo na meia-idade: Do mito à realidade [Subjective well-being in middle age: From myth to reality]. Revista Portuguesa de Pedagogia, 1(31), 149-170.

Mackinnon, A., Jorm, A. F., Christensen, H., Korten, A. E., Jacomb, P. A., \& Rodgers, B. (1999). A short form of the Positive and Negative Affect Schedule: Evaluation of factorial validity and invariance across demographic variables in a community sample. Personality and Individual Differences, 27(3), 405-416. https://doi.org/10.1016/S0191-8869(98)00251-7

Nolla, M. del C., Queral, R., \& Miró, J. (2014). Las escalas PANAS de afecto positivo y negativo: Nuevos datos de su uso en personas mayores [The Positive and Negative Affect Schedule: Further examination of the questionnaire when used with older patients]. Revista de Psicopatología y Psicología Clínica, 19(1), 15-7.

Oliveira, S. F., Queiroz, M. I. N., \& Costa, M. L. A. (2012). Bem estar subjetivo na terceira idade [Subjective wellbeing in old age]. Motricidade, 8(2), 1038-1047. Retrieved from https://www.redalyc.org/pdf/2730/273023568131.pdf

Pearson, L. C., \& Hall, B. W. (1993). Initial construct validation of the teaching autonomy scale. The Journal of Educational Research, 86(3), 172-178. https://doi.org/10.1080/00220671.1993.9941155

Pires, P., Filgueiras, A., Ribas, R., \& Santana, C. (2013). Positive and Negative Affect Schedule: Psychometric properties for the Brazilian Portuguese version. The Spanish Journal of Psychology, 16, e58, 1-9. https://doi.org/10.1017/sjp.2013.60

Runcan, P.-L. (2012). Elderly institutionalization and depression. Procedia - Social and Behavioral Sciences, 33, 109-113. https://doi.org/10.1016/j.sbspro.2012.01.093

Sandín, B., Chorot, P., Lostao, L., Joiner, T. E., Santed, M. A., \& Valiente, R. M. (1999). Escalas PANAS de afecto positivo y negativo: Validación factorial y convergencia transcultural [PANAS Scales of Positive and Negative Affection: Factorial Validation and Cross-Cultural Convergence]. Psicothema, 11(1), 37-51. Retrieved from http://www.psicothema.com/psicothema.asp?id=229

Simões, A. (1992). Ulterior validação de uma escala de satisfação com a vida (SWLS) [Further validation of a life satisfaction scale (SWLS)]. Revista Portuguesa de Pedagogia, 26(3), 503-515.

Simões, A. (1993). São os homens mais agressivos que as mulheres? Revista Portuguesa de Pedagogia, 27(3), 387-404.

Sousa, L. M., Marques-Vieira, C. M. A., Severino, S., Pozo Rosado, J., \& José, H. (2016). Validação da Positive And Negative Affect Schedule em pessoas com doença renal crônica [Positive and Negative Affect Schedule validation in people with chronic kidney disease]. Texto \& Contexto Enfermagem, 25(4), 1-8. https://doi.org/10.1590/0104-07072016005610015

Sposito, G., Diogo, M. J. D., Cintra, F. A., Neri, A. L., \& Guariento, M. E. (2010). Relações entre bem-estar subjetivo e mobilidade e independência funcional por função de grupo de faixas etárias e de gêneros em idosos. Acta Fisiatra, 17(3), 103-108. Retrieved from http://www.revistas.usp.br/actafisiatrica/article/view/103339

Thompson, E. R. (2007). Development and validation of an internationally reliable short-form of the Positive and Negative Affect Schedule (PANAS). Journal of Cross-Cultural Psychology, 38(2), 227-242. https://doi.org/10.1177/0022022106297301 
Vicente, F., Espirito-Santo, H., Cardoso, D., Silva, F. da, Costa, M., Martins, S., ... Lemos, L. (2014). Estudo longitudinal dos fatores associados à evolução de sintomas depressivos em idosos institucionalizados [Longitudinal study of factors associated with the evolution of depressive symptoms in institutionalized elderly]. Jornal Brasileiro de Psiquiatria, 63(4), 308-316. https://doi.org/10.1590/0047-2085000000039

Watson, D., Clark, L. A., \& Tellegen, A. (1988). Development and validation of brief measures of positive and negative affect: The PANAS scales. Journal of Personality and Social Psychology, 54(6), 1063-1070. https://doi.org/10.1037//0022-3514.54.6.1063

Wilson, K., Gullone, E., \& Moss, S. (1998). The youth version of the Positive and Negative Affect Schedule: A psychometric validation. Behaviour Change, 15(3), 187-193. https://doi.org/10.1017/S0813483900003077

Yesavage, J. A., Brink, T. L., Rose, T. L., Lum, O., Huang, V., Adey, M., \& Leirer, V. O. (1983). Development and validation of a geriatric depression screening scale: A preliminary report. Journal of Psychiatric Research, 17(1), 37-49. https://doi.org/10.1016/0022-3956(82)90033-4 